\title{
El color como indicador de contaminación por metales pesados en suelos de la Ciudad de México
}

\section{Color as a proxy for heavy metal pollution in soils of Mexico City}

Francisco Bautista $^{1, *}$, Avto Goguitchaichvili², Carmen Delgado ${ }^{2}$, Patricia Quintana ${ }^{3}$, Daniel Aguilar ${ }^{3}$, Rubén Cejudo ${ }^{2}$, José Luis Cortés ${ }^{1}$

${ }^{1}$ Laboratorio Universitario de Geofísica Ambiental, Centro de Investigaciones en Geografia Ambiental, Universidad Nacional Autónoma de México.

${ }^{2}$ Laboratorio Universitario de Geofisica Ambiental, Instituto de Geofísica, Universidad Nacional Autónoma de México.

${ }^{3}$ Laboratorio Nacional de Nano y Biomateriales, Centro de Investigacionesy Estudios Avanzados.

* Autor para correspondencia: (F. Bautista) leptosol@ciga.unam.mx

\section{RESUMEN}

El objetivo fue la evaluación del color como técnica rápida para la identificación de muestras de suelos urbanos contaminados con metales pesados en la Ciudad de México. Las concentraciones de los metales pesados se midieron por fluorescencia de rayos $\mathrm{X}$, y el color se determinó con las tablas Munsell y con un colorímetro. A partir de los valores Hue, Value y Croma se formaron cinco grupos de suelo por color: I, Café rojizo oscuro, 5YR 4/2; II, Gris oscuro, 7.5YR 4/1; III, Gris olivo, 5Y 5/2; IV, Gris, 5 YR 5/1; V, Gris rosáceo, 7.5YR 6/2. Con los valores colorimétricos en el sistema Rojo, Verde y Azul (RGB) se calcularon tres índices de color: Índice Hue, Índice de Rojez e Índice de saturación. La validación de los grupos de suelo formados con las tablas Munsell fue mayor con los índices de color que con los metales pesados. Los grupos de muestras de suelo urbano I y II de colores oscuros tuvieron las concentraciones mayores del $\mathrm{Cr}, \mathrm{Cu}, \mathrm{Pb}$, $\mathrm{Zn}$; también tuvieron los más altos porcentajes de $\mathrm{Fe}_{2} \mathrm{O}_{3}, \mathrm{MnO}$, y $\mathrm{TiO}_{2}$. Por el contrario, los grupos IV y V con colores claros tuvieron los porcentajes mayores de $\mathrm{Al}_{2} \mathrm{O}_{3}, \mathrm{MgO}_{\text {y }} \mathrm{SiO}_{2}$. El grupo III tuvo valores intermedios de $\mathrm{Cr}, \mathrm{Cu}, \mathrm{Pb}, \mathrm{Zn}$, $\mathrm{Fe}_{2} \mathrm{O}_{3}, \mathrm{MnO}$, y $\mathrm{TiO}_{2}$. Por primera vez se reporta que el color del suelo urbano de la Giudad de México puede ser utilizado como técnica de análisis rápido para la identificación de sitios contaminados con metales pesados.

Palabras clave: color Munsell, sistema RGB, índices de color, técnicas proxy, metales pesados.

\section{ABSTRACT}

The objective was the evaluation of color as a proxy for soil contamination by heavy metals in Mexico City. The concentrations of heavy metals were measured by X-ray fluorescence, and the color was determined with the Munsell Tables and colorimeter using Red Green Blue (RGB) system. From the Hue, Value and Croma values, five soil groups were formed by color: I, Dark reddish brown, 5YR 4/2; II, Dark gray, 7.5YR 4/1; III, Olive gray, 5 Y 5/2; IV, Gray, 5YR 5/1; V, Pinkish gray, 7.51R 6/2. With the Red Green Blue colorimetric values, three color indexes were calculated: Hue index, Redness index and saturation index. Validation of soil groups was greater with color indexes than with heavy metals. Groups I and II of dark color had the highest concentrations of $\mathrm{Cr}, \mathrm{Cu}, \mathrm{Pb}$ and $\mathrm{Zn}$; they also had the highest percentages of $\mathrm{Fe}_{2} \mathrm{O}_{3}, \mathrm{MnO}$, and $\mathrm{TiO}_{2}$. Light colored groups $\mathrm{IV}$ and $\mathrm{V}$ had the highest percentages of $\mathrm{Al}_{2} \mathrm{O}_{3}, \mathrm{MgO}$ and $\mathrm{SiO}_{2}$. Group III had intermediate values of $\mathrm{Cr}, \mathrm{Cu}, \mathrm{Pb}, \mathrm{Zn}, \mathrm{Fe}_{2} \mathrm{O}_{3}, \mathrm{MnO}$, and $\mathrm{TiO}_{2}$. For the first time, it is reported that the color of the urban soil of Mexico City can be used as a rapid analysis technique to identify sites contaminated with heavy metals.

Keywords: color Munsell, RGB system, color indexes, proxy techniques, heavy metals.
La revisión por pares es responsabilidad de la Universidad Nacional Autónoma de México. 


\section{Introducción}

En la Ciudad de México y área conurbada se liberan al ambiente partículas que contienen metales pesados y óxidos de hierro, manganeso, aluminio y silicio que son fácilmente transportadas por el viento, se depositan en diversas superficies como el suelo, y pueden ser inhaladas o ingeridas causando daños a la salud (Sabath y Robles-Osorio, 2012; WHO, 2014; Aguilera et al., 2020).

Las partículas contaminantes que más dañan a la salud humana, y causan cáncer, son menores a 10 micras $\left(\mathrm{PM}_{10}, \mathrm{PM}_{2.5}, \mathrm{PM}_{0.1}\right)$. Su dispersión fuera de la Ciudad de México y área conurbada se retrasa debido a las características climáticas, geológicas, de relieve y dirección de los vientos en la cuenca (Vallejo et al., 2003).

Sus fuentes son naturales, como las emisiones volcánicas, y antrópicas derivadas de las actividades de 23 millones de habitantes, 40000 industrias y la circulación de 4 millones de vehículos (Molina et al., 2010; Ramírez y Martínez, 2018).

Las estrategias de disminución de la contaminación requieren de monitoreos del contenido de metales pesados en muestras ambientales como los realizados por Vallejo et al. (2003) y Molina et al. (2010) en partículas aéreas; Cejudo et al. (2015), Ihl et al. (2015) en suelos y en plantas.

Sin embargo, el monitoreo de la contaminación por metales pesados no se realiza en ningún país del mundo. Esto sucede porque el muestreo de suelos, el tratamiento de la muestra (secado, tamizado y digestión) y los análisis químicos son costosos, consumen mucho tiempo y se generan residuos peligrosos.

Por otro lado, las investigaciones recientes han demostrado que el uso del color puede ser considerado una técnica rápida para inferir la contaminación por metales pesados en polvo urbano (Cortés et al., 2015; García et al., 2019; Marín et al., 2019), así como también en la cantidad de materia orgánica edáfica (Dobos et al., 1990; Schulze et al., 1993; Bautista et al., 2003; Domínguez et al., 2012) y contenido de óxidos de Fe en el suelo (Curi y Franzmeier, 1984; Madeira et al., 1997; AcevedoSandoval et al., 2004; Levin et al., 2005; Viscarra et al., 2008; Kumaravel et al., 2009).
El uso del color como técnica rápida considera que bajo cualquier combustión los óxidos de hierro y la materia orgánica se transforman en magnetita y carbón, ambos son de color negro. La magnetita va acompañada de metales pesados (Bautista et al., 2014; Cortés et al., 2015; García et al., 2019).

El uso del color del suelo como técnica de diagnóstico rápido permitiría seleccionar las muestras de suelo más contaminadas por metales pesados que luego tendrían que pasar por las técnicas de análisis químico convencional (Espectroscopía de Absorción Atómica, ICP-Masas, Microscopía Electrónica de Barrido, entre otros).

El objetivo de este estudio fue la evaluación del color en muestras de suelo de la Ciudad de México y área conurbada como una técnica rápida en el monitoreo de la contaminación del suelo urbano por metales pesados.

\section{Materiales y métodos}

\subsection{ZONA DE ESTUDIO, MUESTREO Y TRATAMIENTO DE LAS MUESTRAS}

El estudio se realizó en la Ciudad de México y área conurbada (Figura 1). La altitud promedio es de 2240 msnm. Geológicamente, está ubicada sobre depósitos volcánicos en el norte, oeste, sur y sureste, y lacustres en la zona central, noreste y sureste (Díaz-Rodríguez, 2006) por lo que es una cuenca rodeada de volcanes (Vallejo et al., 2003).

El diseño del muestreo de suelo fue sistemático y abarcó un área de $1600 \mathrm{~km}^{2}$ dividida en una rejilla de $10 \times 10$. Se recolectaron 89 muestras durante la estación seca (marzo-abril) con la finalidad de que el tiempo de pretratamiento (secado) fuera el menor.

La colecta de las muestras fue con un tubo de policloruro de vinilo de $100 \mathrm{~cm}^{3}$ que se introdujo al suelo.

\subsection{ANÁLISIS QUíMICOS}

Las muestras se secaron durante dos semanas bajo la sombra y a temperatura ambiente; se molieron y se tamizaron con malla de $2 \mathrm{~mm}$; la porción 

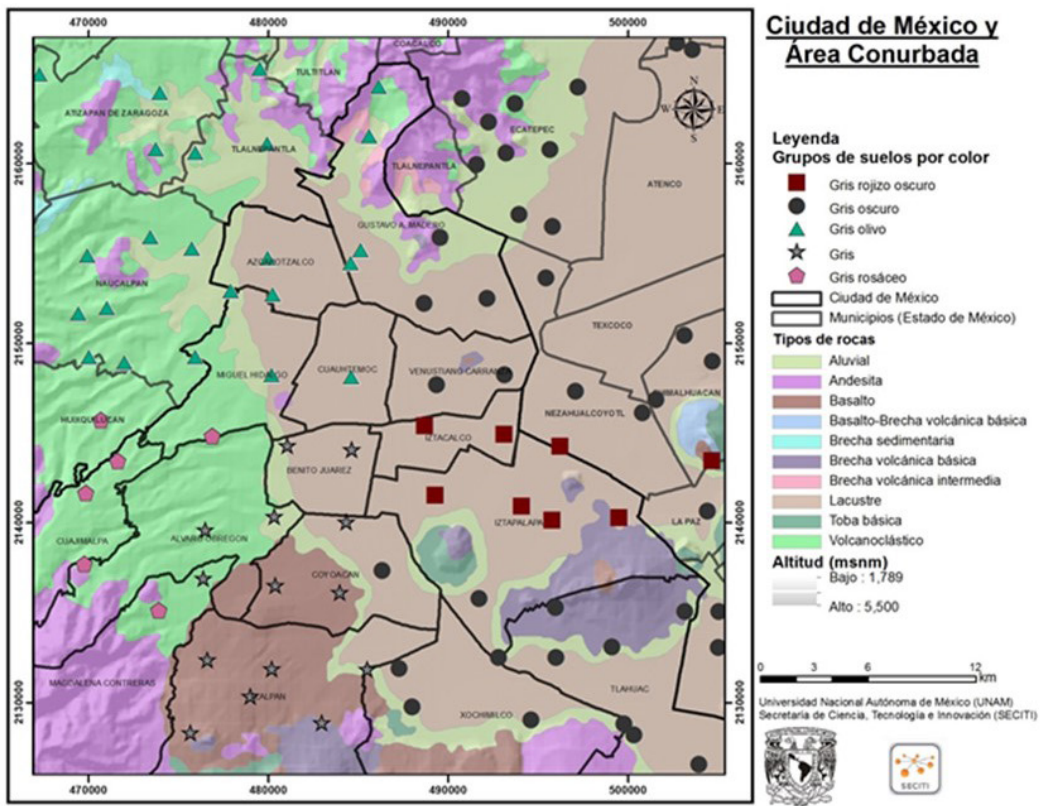

Figura 1 Localización geográfica de las muestras del suelo urbano por grupos de color en la Ciudad de México y área conurbada.

tamizada se dividió en dos partes, una de ellas se utilizó para realizar las mediciones de color y la otra para los análisis geoquímicos.

Las porciones destinadas a los análisis geoquímicos se molieron de nuevo con un mortero de ágata para aumentar la homogeneidad de la muestra (Fitton, 1997; Wei y Yang, 2010; Khodeir et al., 2012; Chen et al., 2014). Las muestras de suelo se comprimieron en un dado de $1 \mathrm{~cm}$ de diámetro sin tratamiento químico o agentes aglutinantes y se obtuvieron pastillas que se colocaron en un porta-muestras y se sellaron con una película de poliéster (Mylar).

Las concentraciones de los metales $(\mathrm{Cr}, \mathrm{Cu}$, $\mathrm{Ni}, \mathrm{Pb}, \mathrm{V}$ y $\mathrm{Zn}$ ) y de los compuestos óxidos metálicos del suelo $\left(\mathrm{Na}_{2} \mathrm{O}, \mathrm{MgO}, \mathrm{MnO}, \mathrm{Al}_{2} \mathrm{O}_{3}, \mathrm{SiO}_{2}\right.$, $\mathrm{K}_{2} \mathrm{O}, \mathrm{CaO}, \mathrm{TiO}_{2}$ y $\left.\mathrm{Fe}_{2} \mathrm{O}_{3}\right)$ y no metálicos $\left(\mathrm{P}_{2} \mathrm{O}_{5}\right)$ se cuantificaron con la técnica de fluorescencia de rayos $\mathrm{X}$ de energía dispersa (ED-FRX) en el Centro de Investigación y de Estudios Avanzados de Mérida, Yucatán. La técnica es cuantitativa, rápida, no destructiva ni contaminante con un límite de detección de $1 \mathrm{mg} / \mathrm{kg}$ (Lozano y Bernal, 2005; Beckhoff et al., 2006). Como control de medición se consideró el estándar con clave de identificación IGLsy-1, el cual corresponde a una sienita de nefelina con un alto contenido de $\mathrm{Al}$ y
Si (Lozano y Bernal, 2005; Beckhoff et al., 2006; Paltridge et al., 2012).

Las características y ambientes de operación del espectrómetro ED-XRF fueron las siguientes: marca Jordan Valley EX-6600, equipado con un detector $\mathrm{Si}(\mathrm{Li})$, área activa de $20 \mathrm{~mm}^{2}$, resolución de $140 \mathrm{eV}$ a $5.9 \mathrm{keV}$, operado a un máximo de 54 keV y $4800 \mu \mathrm{A}$, en condiciones de vacío atmosférico, utilizando una tarjeta secundaria intercambiable y tiempo de medición de 300 s. Cada medición fue repetida cinco veces para obtener un promedio de concentración (Ihl et al., 2015).

\subsection{DETERMINAGIÓN DEL GOLOR}

El color del suelo se determinó de manera cualitativa por comparación con las tablas Munsell (Munsell Color, 2000). La muestra seca se colocó en el porta-muestras, y en un ambiente externo y con luz del día se sobrepusieron a la muestra, las cartas del color hasta encontrar el matiz o tono adecuado. Los suelos se agruparon de acuerdo con el color definido por los valores Hue, Value y Croma.

El color se determinó de manera cuantitativa con un equipo Konica Minolta modelo CR400m, los resultados, en términos del sistema de color X, 
Y, Z se convirtieron al sistema R, G, B decimal mediante el programa Color Slide Rule y se calcularon los índices de color Hue (IH), Rojez (IR) y Saturación (IS) según Levin et al., (2005) de la manera siguiente:

$$
\begin{aligned}
& I H=\frac{(2 * R-G-B)}{(G-B)} \\
& I R=\frac{R^{2}}{\left(B^{*} G^{3}\right)} \\
& I S=\frac{(R-B)}{(R+B)}
\end{aligned}
$$

En donde R, G y B son los valores proporcionados por el sistema decimal RGB: $\mathrm{R}=$ Rojo; $\mathrm{G}=$ Verde; B = Azul.

\subsection{ANÁLISIS DE DATOS}

La influencia de los metales pesados en los índices de color se evaluó mediante un análisis de correlación simple.

Los cinco grupos de color del suelo identificados a partir de la comparación con las tablas Munsell, se validaron mediante dos análisis discriminantes: por un lado, con los valores de los contenidos de metales pesados y óxidos, y por otro, con los valores de los índices de color en donde se clasificaron mediante funciones lineales, que combinan las variables más influyentes y asignan los elementos a los grupos predefinidos, lo que permitió validar o no la formación de los grupos de suelo (Lévy et al., 2003). También se midió la influencia del conjunto de los metales pesados en cada índice de color al interior de cada grupo de color de suelo a través de análisis de regresión múltiple.

En ambos análisis las variables dependientes fueron los índices de color IH, IR e IS y las variables independientes fueron las concentraciones de $\mathrm{Cr}, \mathrm{Ni}, \mathrm{Cu}, \mathrm{Zn}, \mathrm{Pb}, \mathrm{V}$ y los porcentajes de los óxidos $\mathrm{Na}_{2} \mathrm{O}, \mathrm{MgO}, \mathrm{Al}_{2} \mathrm{O}_{3}, \mathrm{SiO}_{2}, \mathrm{P}_{2} \mathrm{O}_{5}, \mathrm{~K}_{2} \mathrm{O}, \mathrm{CaO}$, $\mathrm{TiO}_{2}, \mathrm{MnO}, \mathrm{Fe}_{2} \mathrm{O}_{3}$.

La regresión lineal múltiple establece la relación de la variable dependiente $\mathrm{Y}$ con respecto de múltiples variables independientes $\mathrm{X}\left(\mathrm{X}_{1}, \mathrm{X}_{2} \ldots \mathrm{X}_{\mathrm{n}}\right)$ en un espacio multidimensional. Se determinaron los valores de las variables con mayor poder explicativo mediante los coeficientes de correlación $\beta_{1}$, $\beta_{2} \ldots \beta_{\mathrm{n}}$ (Vallejo, 2011).

$$
\mathrm{Y}=\mathrm{a}+\beta_{1} \mathrm{X}_{1}+\beta_{2} \mathrm{X}_{2}+\ldots \beta_{\mathrm{n}} \mathrm{X}_{\mathrm{n}}+\mathrm{e}
$$

En donde: $\mathrm{Y}=$ variable dependiente; $\mathrm{a}=$ parámetro de regresión; $\beta_{1}=$ incremento de $\mathrm{Y}$ en unidades al aumentar $\mathrm{X} ; \mathrm{X}_{\mathrm{i}}=$ variables explicativas; $\mathrm{e}=$ error de la predicción.

Para comparar los contenidos de los metales pesados por los grupos de color de los suelos se utilizó el análisis de varianza no paramétrico de comparación de medianas Kruskal-Wallis (Statgraphics®, 2010). Se aplicó una prueba de rango múltiple para determinar qué grupos de suelo por color son significativamente diferentes con un nivel de confianza del 95.0\%. Se diseñó un mapa con las diferentes zonas donde se ubicaron los cinco grupos de color del suelo y los tipos de rocas subyacentes con el programa ArcGIS 9.0 (ESRI, 2004).

\section{Resultados}

\subsection{FORMAGIÓN Y VALIDAGIÓN DE LOS GRUPOS POR GOLOR DEL SUELO}

Se formaron cinco grupos por color del suelo. Los grupos I Gris rojizo oscuro y II Gris oscuro presentaron coloraciones oscuras, mientras que los suelos del grupo III Gris olivo, grupo IV Gris y el grupo V Gris rosáceo presentaron las coloraciones claras (Tabla 1, Figura 2).

La validación de los grupos por color del suelo utilizando los índices tuvo una clasificación correcta del $100 \%$ de las muestras en todos los grupos de suelo $(\mathrm{p}<0.05)$ (Tabla 2). La validación de los grupos por color del suelo utilizando las concentraciones de los metales pesados tuvieron un $67 \%$ de las muestras correctamente asignadas, siendo los grupos I y II los de mayor porcentaje de correcta asignación, con $88 \%$ y $68 \%$, respectivamente (Tabla 3). En análisis multivariados los porcentajes arriba del 50\% son aceptables (Lévy et al., 
Tabla 1. Agrupación de las muestras de suelo por color a partir de los valores hue, value y croma identificados con las tablas Munsell.

\begin{tabular}{|l|c|c|c|c|c|}
\hline Grupo de color & Muestras (n) & Hue & Value & Croma & Notación \\
\hline I Gris rojizo oscuro & 8 & $5 Y R$ & 4 & 2 & 5 YR 4/2 \\
\hline II Gris oscuro & 38 & $7.5 Y R$ & 4 & 1 & 7.5 YR 4/1 \\
\hline III Gris olivo & 23 & $5 Y$ & 5 & 2 & $5 Y$ Y 5/2 \\
\hline IV Gris & 14 & $5 Y R$ & 5 & 1 & 5 YR 5/1 \\
\hline V Gris rosáceo & 6 & $7.5 Y R$ & 6 & 2 & $7.5 Y R 6 / 2$ \\
\hline
\end{tabular}

Tabla 2. Validación de los grupos de color del suelo con base en los índices de color hue (IH), rojez (IR) y saturación (IS).

\begin{tabular}{|l|c|c|c|c|c|c|}
\hline \multirow{2}{*}{ Color real } & \multirow{2}{*}{$\begin{array}{c}\text { Muestras } \\
\text { (n) }\end{array}$} & I Gris rojizo & II Gris & III Gris & IV & V Gris \\
\cline { 3 - 7 } & obscuro & obscuro & olivo & Gris & rosáceo \\
\hline I Gris rojizo oscuro & 8 & $8(100 \%)$ & $0(0 \%)$ & $0(0 \%)$ & $0(0 \%)$ & $0(0 \%)$ \\
\hline II Gris oscuro & 38 & $0(0 \%)$ & $38(100 \%)$ & $0(0 \%)$ & $0(0 \%)$ & $0(0 \%)$ \\
\hline III Gris olivo & 23 & $0(0 \%)$ & $0(0 \%)$ & $23(100 \%)$ & $0(0 \%)$ & $0(0 \%)$ \\
\hline IV Gris & 14 & $0(0 \%)$ & $0(0 \%)$ & $0(0 \%)$ & $14(100 \%)$ & $0(0 \%)$ \\
\hline V Gris rosáceo & 6 & $0(0 \%)$ & $0(0 \%)$ & $0(0 \%)$ & $0(0 \%)$ & $6(100 \%)$ \\
\hline
\end{tabular}

Tabla 3. Validación de los grupos de color del suelo con base en las concentraciones de los metales pesados.

\begin{tabular}{|c|c|c|c|c|c|c|}
\hline \multirow[b]{2}{*}{ Grupos } & \multirow{2}{*}{$\begin{array}{c}\text { Muestras } \\
\text { (n) }\end{array}$} & \multicolumn{5}{|c|}{$\begin{array}{l}\text { Color estimado a partir de las concentraciones de los metales } \\
\qquad \text { pesados }\end{array}$} \\
\hline & & $\begin{array}{c}\text { I Gris rojizo } \\
\text { obscuro }\end{array}$ & $\begin{array}{l}\text { II Gris } \\
\text { obscuro }\end{array}$ & $\begin{array}{c}\text { III Gris } \\
\text { olivo }\end{array}$ & $\begin{array}{c}\text { IV } \\
\text { Gris }\end{array}$ & $\begin{array}{l}\text { V Gris } \\
\text { rosáceo }\end{array}$ \\
\hline $\begin{array}{l}\text { I Gris rojizo } \\
\text { oscuro }\end{array}$ & 8 & $7(88 \%)$ & $0(0 \%)$ & $1(12 \%)$ & $0(0 \%)$ & $0(0 \%)$ \\
\hline II Gris oscuro & 38 & $3(8 \%)$ & $26(68 \%)$ & $6(16 \%)$ & $1(3 \%)$ & $2(5 \%)$ \\
\hline III Gris olivo & 23 & $0(0 \%)$ & $5(22 \%)$ & $15(65 \%)$ & $1(4 \%)$ & $2(9 \%)$ \\
\hline IV Gris & 14 & $0(0 \%)$ & $2(14 \%)$ & $1(7 \%)$ & $9(64 \%)$ & $2(14 \%)$ \\
\hline V Gris rosáceo & 6 & $0(0 \%)$ & $0(0 \%)$ & $1(17 \%)$ & $2(33 \%)$ & $3(50 \%)$ \\
\hline
\end{tabular}


2003). La medición del color con un colorímetro es más rápida, cuantitativa y precisa, mientras que con las tablas Munsell es más tardada, cualitativa y menos precisa pero el análisis es más económico. La validación al 100\% de los grupos con los índices de color indica que las mediciones colorimétricas son confiables con ambos métodos.

\subsection{REGRESIONES LINEALES MÚLTIPLES ENTRE LOS ÍNDIGES DE GOLOR DEL SUELO Y LAS CONCENTRACIONES DE METALES PESADOS}

La relación ente los metales pesados con los tres índices de color fue alta y significativa en los grupos de color I Gris rojizo obscuro, IV Gris y V Gris rosáceo $\left(\mathrm{r}^{2}>0.75, \mathrm{p}<0.05\right)$. Con el índice Hue (IH) los metales pesados mejor representados en los cinco grupos de suelos fueron el $\mathrm{Cr}, \mathrm{Cu}$ y $\mathrm{Pb}$, en menor proporción $\mathrm{Ni}, \mathrm{V}$, así como $\mathrm{MnO}$ y $\mathrm{Fe}_{2} \mathrm{O}_{3}$ (Tabla 4); con el índice de rojez (IR) fueron el $\mathrm{Cr}, \mathrm{Ni}, \mathrm{Pb}$ y $\mathrm{Cu}$, en menor proporción el $\mathrm{V}, \mathrm{MnO}$ y $\mathrm{Fe}_{2} \mathrm{O}_{3}$ y $\mathrm{Zn}$ (Tabla 5); y con el índice de saturación (IS) fueron el $\mathrm{Cr}, \mathrm{Cu}, \mathrm{Ni}$ y $\mathrm{Pb}$, en menor proporción el $\mathrm{V}$ y $\mathrm{Zn}$ así como $\mathrm{MnO}$ y $\mathrm{Fe}_{2} \mathrm{O}_{3}$ (Tabla 6). En total los metales pesados representados en los cinco grupos de suelos con los tres índices de color tuvieron el siguiente orden: $\mathrm{Cr}=\mathrm{Pb}>\mathrm{Cu}>\mathrm{Ni}=\mathrm{V}>\mathrm{MnO}>\mathrm{Fe}_{2} \mathrm{O}_{3}>\mathrm{Zn}$.

\subsection{FORMAGIÓN Y VALIDAGIÓN DE LOS GRUPOS POR GOLOR DEL SUELO}

Las concentraciones mayores $(\mathrm{p}<0.05)$ de los metales $\mathrm{Cr}, \mathrm{Cu}, \mathrm{Pb}$ y $\mathrm{Zn}$ se encontraron en los grupos I y II de color oscuro; y las concentraciones medias en el grupo III de coloración intermedia entre color oscuro y claro (Figuras 2 y 3). Los porcentajes mayores del $\mathrm{Fe}_{2} \mathrm{O}_{3}, \mathrm{MnO}$, y $\mathrm{TiO}_{2}$ se encontraron en los grupos oscuros I y II de color oscuro (Figuras 2 y 4); y los mayores porcentajes de los óxidos $\mathrm{Al}_{2} \mathrm{O}_{3}, \mathrm{MgO}$ y $\mathrm{SiO}_{2}$ se encontraron principalmente en los grupos III, IV y V de coloraciones claras (Figuras 2 y 4). En los grupos I y II la presencia de los metales $\mathrm{Cr}, \mathrm{Cu}, \mathrm{Zn}, \mathrm{Fe}, \mathrm{Mn}$ y Ti les confieren las coloraciones más oscuras, Domínguez et al. (2012) reporta que los metales de transición producen coloraciones oscuras.

\subsection{LOGALIZAGIÓN GEOGRÁFICA DE LAS MUESTRAS DE SUELO POR GOLOR}

Las muestras de suelo del grupo I Gris rojizo obscuro se ubican principalmente en la zona Lacustre en el centro-este de la ciudad, en las delegaciones Iztacalco e Iztapalapa y en las zonas conurbadas de Cd. Netzahualcóyotl, Chimalhuacán y La Paz (Figura 1). Este grupo en general tiene las

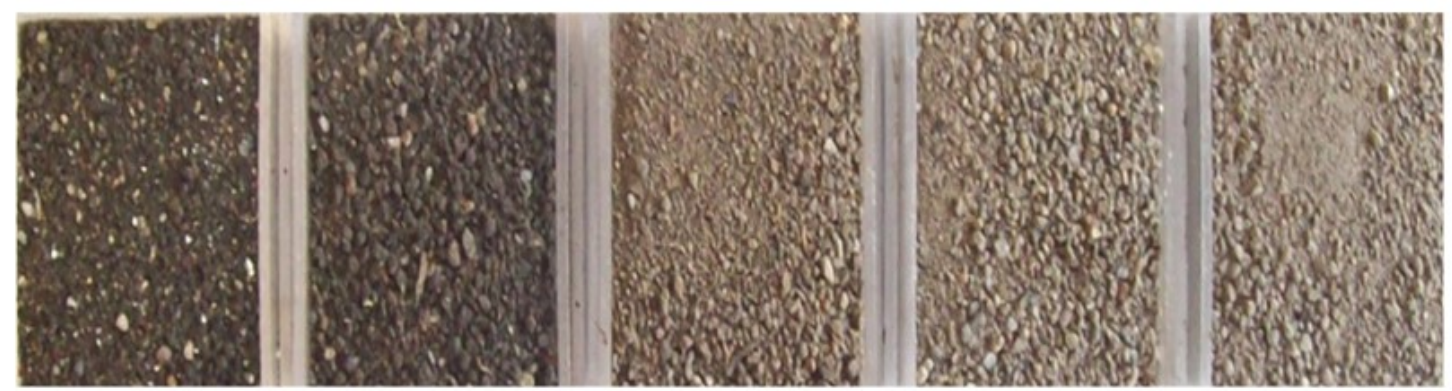

Gris rojizo Gris oscuro Gris olivo Gris $\quad$ Gris rosáceo

$\begin{array}{lllll}\text { oscuro } & 7.5 \mathrm{YR} 4 / 1 & 5 \mathrm{Y} 5 / 2 & 5 \mathrm{YR} 5 / 1 & 7.5 \mathrm{YR} 6 / 2\end{array}$

\section{$5 \mathrm{YR} 4 / 2$}


Tabla 4. Regresiones lineales múltiples entre el índice hue (IH) y las concentraciones de los metales pesados en los grupos de color de suelo.

\begin{tabular}{|l|l|c|c|}
\hline Grupos & \multicolumn{1}{|c|}{ Ecuación de regresión } & $\mathrm{r}^{2}$ & Valor p \\
\hline $\begin{array}{l}\text { I Gris rojizo } \\
\text { oscuro }\end{array}$ & $\begin{array}{l}\mathrm{IH}=-159.06-0.08 * \mathrm{Cr}-0.28 * \mathrm{Ni}+0.19 * \mathrm{Cu}+0.10 * \mathrm{Zn}- \\
0.03 * \mathrm{~Pb}+1.20 * \mathrm{~V}+294.09 * \mathrm{MnO}\end{array}$ & 0 \\
\hline II Gris oscuro & $\mathrm{IH}=1.38+0.05 * \mathrm{Cr}-0.02 * \mathrm{Cu}+0.53 * \mathrm{Fe}_{2} \mathrm{O}_{3}+0.003 * \mathrm{~Pb}$ & 0.21 & 0.09 \\
\hline III Gris olivo & $\mathrm{IH}=11.38-0.01 * \mathrm{Cr}+0.001 * \mathrm{Cu}-31.28 * \mathrm{MnO}+0.004 * \mathrm{Ni}$ & 0.16 & 0.69 \\
\hline IV Gris & $-0.004 * \mathrm{~Pb}$ & \\
\hline & $\mathrm{IH}=7.8-0.04 * \mathrm{Cr}+0.01 * \mathrm{Cu}-0.59 * \mathrm{Fe}_{2} \mathrm{O}_{3}+21.91 * \mathrm{MnO}-$ & 0.83 & 0.04 \\
\hline V Gris rosáceo & $\mathrm{IH}=19.28-0.04 * \mathrm{Cr}-0.01 * \mathrm{Cu}-0.01 * \mathrm{Ni}-0.002 * \mathrm{~Pb}-$ & 1 \\
\hline & $0.04 * \mathrm{~V}$ & 0 \\
\hline
\end{tabular}

Tabla 5. Regresiones lineales múltiples entre el índice rojez (IR) y las concentraciones de los metales pesados en los grupos de color de suelo.

\begin{tabular}{|c|c|c|c|}
\hline Grupos & Ecuación de regresión & $r^{2}$ & Valor $\mathrm{p}$ \\
\hline $\begin{array}{l}\text { I Gris rojizo } \\
\text { oscuro }\end{array}$ & $\begin{array}{l}\mathrm{IR}=-0.05+0.00008 * \mathrm{Cr}-0.0003 * \mathrm{Ni}-0.0001 * \mathrm{Cu}+0.0001 * \mathrm{Zn}- \\
0.00002 * \mathrm{~Pb}+0.0004 * \mathrm{~V}+0.06 * \mathrm{MnO}\end{array}$ & 1 & 0 \\
\hline II Gris oscuro & $\begin{array}{l}\mathrm{IR}=0.008+0.02 * \mathrm{MnO}-0.000008 * \mathrm{Cr}-0.000004 * \mathrm{Cu}- \\
0.001 * \mathrm{Fe}_{2} \mathrm{O}_{3}-0.000006 * \mathrm{Ni}+0.000003 * \mathrm{~Pb}\end{array}$ & 0.33 & 0.04 \\
\hline III Gris olivo & $\begin{array}{l}\mathrm{IR}=0.003-0.000001 * \mathrm{Cr}+0.000002 * \mathrm{Cu}+0.000004 * \mathrm{Ni}- \\
0.000002 * \mathrm{~Pb}\end{array}$ & 0.08 & 0.4 \\
\hline IV Gris & $\begin{array}{l}\mathrm{IR}=-0.005-0.000006 * \mathrm{Cr}+0.0004 * \mathrm{Fe}_{2} \mathrm{O}_{3}+0.000002 * \mathrm{Ni}- \\
0.000002 * \mathrm{~Pb}+0.00006 * \mathrm{~V}\end{array}$ & 0.75 & 0.04 \\
\hline $\begin{array}{l}\text { V Gris } \\
\text { rosáceo }\end{array}$ & $\begin{array}{l}\mathrm{IR}=-0.02+0.0001 * \mathrm{Cr}-0.00002 * \mathrm{Cu}+0.00001 * \mathrm{Ni}+ \\
0.000003 * \mathrm{~Pb}+0.00008 * \mathrm{~V}\end{array}$ & 1 & 0 \\
\hline
\end{tabular}

mayores concentraciones de $\mathrm{Cu}, \mathrm{Pb}, \mathrm{Zn}, \mathrm{Fe}_{2} \mathrm{O}_{3}$, $\mathrm{MnO}$ y $\mathrm{TiO}_{2}$. Las muestras de suelo del grupo II Gris oscuro están ubicadas principalmente en la zona Lacustre en las alcaldías Gustavo A. Madero, Venustiano Carranza, Coyoacán, Iztapalapa, Xochimilco y Tláhuac, así como en Cd. Netzahualcóyotl, Chimalhuacán, La Paz Tlalnepantla y Ecatepec (Figura 1). Este grupo en general tiene las más altas concentraciones de $\mathrm{Cr}$, y concentraciones altas de $\mathrm{Cu}, \mathrm{Pb}, \mathrm{Zn}$, $\mathrm{Fe}_{2} \mathrm{O}_{3}, \mathrm{MnO}_{\text {y }} \mathrm{TiO}_{2}$. Las muestras de suelo del grupo III Gris olivo se localizan principalmente en zonas conurbadas de Atizapán de Zaragoza, Naucalpan y en Tlalnepantla; así como en las alcaldías de Cuauhtémoc, Azcapotzalco, Gustavo A. Madero y Miguel Hidalgo (Figura 1). Este grupo en general tiene las concentraciones más altas de $\mathrm{SiO}_{2}$ y concentraciones medias de $\mathrm{Cr}, \mathrm{Cu}$, $\mathrm{Pb}, \mathrm{Zn}$ y $\mathrm{Al}_{2} \mathrm{O}_{3}$. Las muestras de suelo de los grupos IV y V se localizan en la periferia de la ciudad, son las muestras con menores concentraciones de metales pesados. 
Tabla 6. Regresiones lineales múltiples entre el índice de saturación (IS) y las concentraciones de los metales pesados en los grupos de color de suelo.

\begin{tabular}{|l|l|c|c|}
\hline Grupos & \multicolumn{1}{|c|}{ Ecuación de regresión } & $\mathrm{r}^{2}$ & Valor p \\
\hline $\begin{array}{l}\text { I Gris rojizo } \\
\text { oscuro }\end{array}$ & $\mathrm{IS}=-0.42+0.005 * \mathrm{Cr}-0.01 * \mathrm{Ni}-0.007 * \mathrm{Cu}+0.004 * \mathrm{Zn}-$ & 1 & 0 \\
\hline $\begin{array}{l}0.0005 * \mathrm{~Pb}+0.003 * \mathrm{~V}+0.36 * \mathrm{MnO} \\
\text { II Gris oscuro }\end{array}$ & $\mathrm{IS}=0.29-0.001 * \mathrm{Cr}-0.01 * \mathrm{Fe}_{2} \mathrm{O}_{3}+0.0001 * \mathrm{~Pb}+0.001 * \mathrm{~V}$ & 0.34 & 0 \\
\hline III Gris olivo & $\mathrm{IS}=0.07-0.0003 * \mathrm{Zn}+0.001 * \mathrm{Ni}+0.0006 * \mathrm{Cu}+1.28 * \mathrm{MnO}$ & 0.34 & 0.09 \\
\hline IV Gris & $\mathrm{IS}=-0.08+0.0008 * \mathrm{Cr}+0.0006 * \mathrm{Cu}+0.05 * \mathrm{Fe}_{2} \mathrm{O}_{3}-0.65 * \mathrm{MnO}$ & 0.95 & 0 \\
\hline & $+0.003 * \mathrm{Ni}+0.00005 * \mathrm{~Pb}-0.0006 * \mathrm{Zn}$ & \\
\hline V Gris & $\mathrm{IS}=-0.03+0.001 * \mathrm{Cr}-0.0002 * \mathrm{Cu}+0.0002 * \mathrm{Ni}+0.0001 * \mathrm{~Pb}+$ & 1 & 0 \\
\hline rosáceo & $0.001 * \mathrm{~V}$ & \\
\hline
\end{tabular}

\section{Discusión}

Las muestras de suelo urbano de los grupos I (gris rojizo oscuro) y II (gris oscuro), y en algunos casos el grupo III (gris olivo), alcanzaron las concentraciones mayores de $\mathrm{Cu}, \mathrm{Pb}$ y $\mathrm{Zn}$ en este estudio, de la misma manera que lo reportado para el polvo urbano en Ensenada Baja California (Cortés et al., 2015). Estos metales pesados se consideran producto de la contaminación antrópica al estar muy por arriba de los valores de fondo, como por ejemplo los $20 \mathrm{mg} / \mathrm{kg}$ de plomo, $36 \mathrm{mg} / \mathrm{kg}$ de zinc, $41 \mathrm{mg} / \mathrm{kg}$ de cromo y $7 \mathrm{mg} / \mathrm{kg}$ de cobre (Ihl et al., 2015).

La presencia del $\mathrm{Fe}_{2} \mathrm{O}_{3}$ en las muestras de suelo urbano de los grupos I, II y III les proporciona las coloraciones oscuras, y se explica por la relación encontrada entre el índice de rojez y el $\mathrm{Fe}_{2} \mathrm{O}_{3}$, por lo que se infiere la presencia de maghemita o magnetita (Bautista et al., 2014).

El índice de rojez del color del suelo ha sido utilizado para estimar la intensidad de meteorización del suelo y los contenidos de $\mathrm{Fe}_{2} \mathrm{O}_{3}$ (Barrón y Torrent 1986; Levin et al., 2005; Viscarra Rossel et al., 2008); sin embargo, al parecer en este estu- dio la relación podría ser con la magnetita y/o maghemita que son producto de la combustión de combustibles fósiles y por lo tanto de carácter antrópico, por los mayores porcentajes de $\mathrm{Fe}_{2} \mathrm{O}_{3}$, $\mathrm{TiO}_{2}$ y $\mathrm{MnO}$ los contenidos en partículas producto de la combustión (Abbott, 1987; Bautista et al., 2014). Para estudios futuros es conveniente medir los parámetros magnéticos y si los valores de la susceptibilidad magnética y de la magnetización remanente isotermal de saturación son también altos, entonces el mineral será magnetita y/o maghemita y, por lo tanto, serán partículas antrópicas contaminantes (Aguilar-Reyes et al., 2013; Bautista et al., 2014; Sánchez-Duque et al., 2015).

En las muestras de suelo urbano de los grupos IV y V las coloraciones claras están asociadas con la predominancia del $\mathrm{Al}_{2} \mathrm{O}_{3}, \mathrm{MgO}$ y $\mathrm{SiO}_{2}$ y con bajas concentraciones de $\mathrm{TiO}_{2}, \mathrm{MnO}$ y $\mathrm{Fe}_{2} \mathrm{O}_{3}$. Además, en estos grupos se presentaron los valores más bajos del índice de rojez. De la misma manera, Domínguez et al. (2012) reportaron la relación entre las coloraciones claras del suelo y la presencia de $\mathrm{SiO}_{2}$ y $\mathrm{Al}_{2} \mathrm{O}_{3}$, asociados a aluminosilicatos y cuarzo, principalmente. 

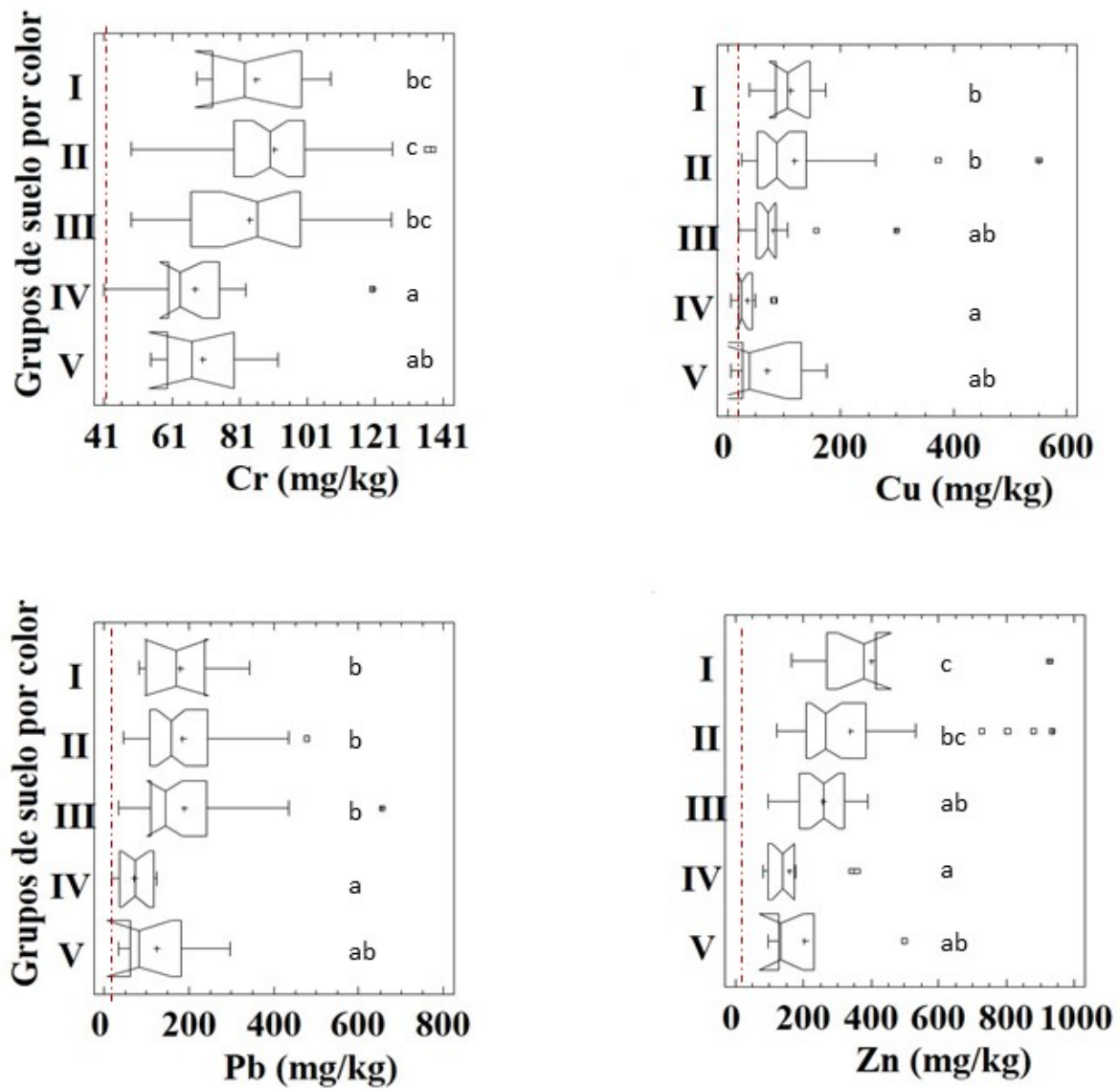

Figura 3 Concentraciones de metales pesados por grupos de color de suelos. I Gris rojizo oscuro, II Gris oscuro, III Gris olivo, IV Gris, $\checkmark$ Gris rosáceo. Línea punteada señala el nivel de fondo. Letras diferentes significan diferencias significativas.

El uso de combustibles fósiles genera ceniza y gases. La ceniza de color negro estaría formada por partículas que contienen óxidos de hierro transformados a magnetita y maghemita (coloraciones obscuras) (Bautista et al., 2014), así como metales pesados usados como antidetonantes $(\mathrm{Mn})$ (Bueno-Brito et al., 2005) o que son parte de las impurezas del combustible (metales pesados, principalmente $\mathrm{Pb}, \mathrm{Zn}, \mathrm{Cu}, \mathrm{V}$ ) (Ihl et al., 2015). Sin embargo, la materia orgánica humificada también es de color negro o pardo obscuro, así como los óxidos de manganeso de color negro (pirolusita), ambos pudieran interferir o confundir cuando se utilice el color como indicador de contaminación por metales pesados en los suelos.

La pirolusita es el mineral de manganeso del color gris a negro que se encuentra naturalmente en las rocas que circundan a la ciudad de México, pero también el manganeso es utilizado como antidetonante en la gasolina (1 metil-4-fenil-1,2,5,6-tetrahidropiridina) y es un componente del acero (Bueno-Brito et al., 2005) y se encuentra en el polvo urbano de la Ciudad de México (Aguilera et al., 2020), así que también tiene un origen antrópico.

\section{Conclusiones}

La principal conclusión de este trabajo fue: el color del suelo urbano puede ser utilizado como técnica de análisis rápido, de bajo costo y con la precisión suficiente para identificar muestras de suelo contaminadas con metales pesados. Es importante hacer notar que este indicador es solamente para suelos urbanos o Tecnosols.

En primera instancia, utilizando el color como técnica rápida para la identificación de meta- 


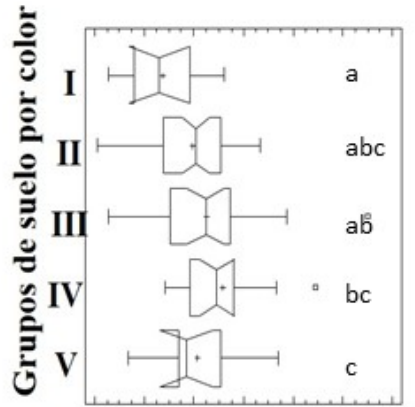

$10141822 \quad 263034$ $\mathrm{Al}_{2} \mathrm{O}_{3}(\%)$

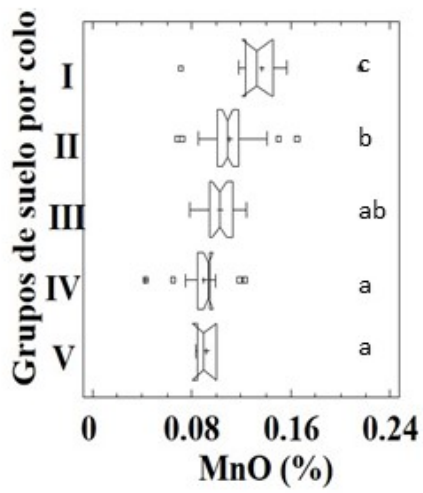

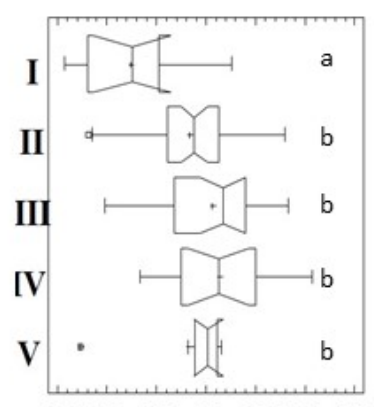

$\begin{array}{lllllll}4752 & 62 & 67 & 72 & 77\end{array}$ $\mathrm{SiO}_{2}(\%)$

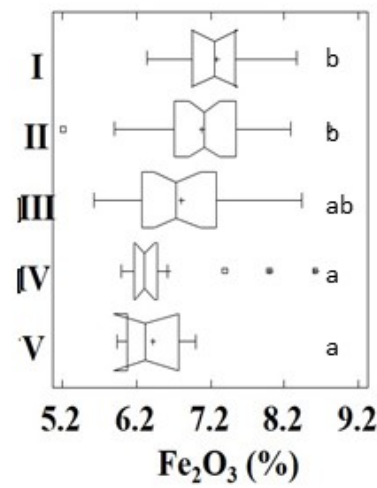

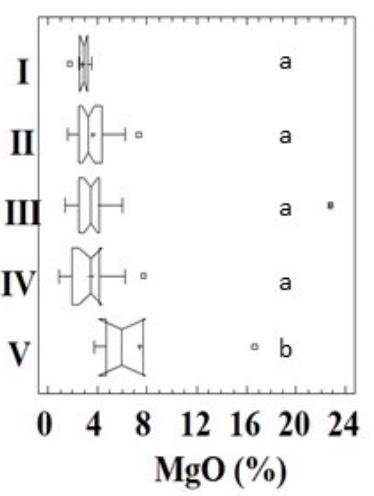

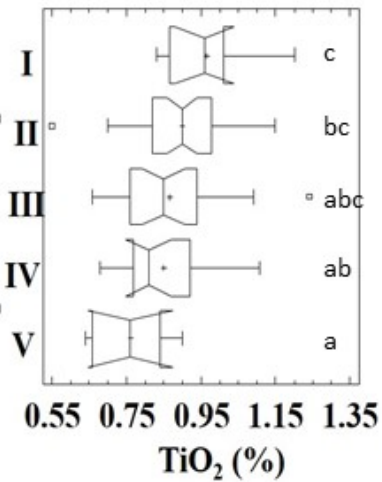

Figura 4 Concentraciones de los óxidos por grupos de color de suelos. I Gris rojizo oscuro, II Gris oscuro, III Gris olivo, IV Gris, V Gris rosáceo. Letras diferentes significan diferencias significativas.

les pesados en el suelo urbano de la Ciudad de México las clases serían: suelos contaminados gris rojizo oscuro 5YR 4/2 y gris oscuro 7.5YR 4/1, contaminación intermedia suelos grises olivo $5 \mathrm{Y}$ 5/2; y suelos poco contaminados los grises $5 \mathrm{YR}$ $5 / 1$ y grises rosáceos $7.5 \mathrm{YR} 6 / 2$.

Los índices de color del suelo urbano solo se validaron, mediante regresiones múltiples, para los suelos de colores gris rojizo oscuro y gris oscuro debido, probablemente, a la interferencia de componentes naturales, como los minerales de hierro y manganeso y a la materia orgánica humificada.

\section{Agradecimientos}

Los autores agradecen el financiamiento al proyecto a la Dirección General de Asuntos del Personal Académico de la Universidad Nacional Autónoma de México (PAPIIT IN 209218) y al CONAGYT (283135).

\section{Referencias}

Abbott,P.J., 1987,Methylcyclopentadienyl manganese tricarbonyl (MMT) in petrol: The toxicological issues: Science of the Total Environment, 67 (2-3), 247-55. https://doi. org/10.1016/0048-9697(87)90215-4.

Acevedo-Sandoval, O., Ortiz-Hernández, E., Cruz-Sánchez, M., Cruz-Chávez, E., 2004, El papel de óxidos de hierro en suelos: Terra Latinoamericana, 22(4), 485-497.

Aguilar-Reyes, B., Mejía, V., Goguichaishvili, A., Escobar, J., Bayona, G., Bautista, F., Morales Contreras, J., Ihl, T., 2013, Reconnaissance environmental magnetic study of urban soils, dust and leaves from Bogotá, Colombia: Studia Geophysica et Geodaetica, 57 (4), 741-754. https://doi.org/10.1007/ s1 1200-012-0682-9.

Aguilera A, Bautista F, Gogichaichvili A, Gutiérrez-Ruiz ME， Ceniceros-Gómez 
AE, López-Santiago NR., 2020, Spatial distribution of manganese concentration and load in street dust in Mexico City: Salud Publica México, 62(2),147-155. https://doi. org/10.21149/10577.

Barrón, V., Torrent, J., 1986, Use of the KubelkaMunk theory to study the influence of iron oxides on soil colour: European Journal of Soil Science, 37, 499-510. https://doi. org/10.1111/j.1365-2389.1986.tb00382.x

Bautista-Zúñiga, F., Jiménez-Osornio, J., Navarro-Alberto, J., Manu, A., Lozano, R., 2003, Microrelieve y color del suelo como propiedades de diagnóstico en Leptosoles cársticos: Terra, 21, 1-11.

Bautista, F., Cejudo, R., Aguilar, B., Goguichaishvili, A., 2014, El potencial del magnetismo en la clasificación de suelos: una revisión: Boletín de la Sociedad Geológica Mexicana, 66 (2), 123-134. http://dx.doi.org/10.18268/ BSGM2014v66n2a11

Beckhoff, B., Kanngießer, B., Langhoff, N., Wedell, R., Wolff, H., 2006, Handbook of practical X-ray fluorescence analysis: Berlin Heidelberg, Springer-Verlag, Alemania, 878 p. https://doi.org/ https://doi.org/ 10.1007/978-3-540-36722-2

Bueno-Brito, C., Sánchez-Ramos, A., ArmentaSolís, A., González-Vera, E.,2005, Contenido de plomo y manganeso en despachadores de gasolina: Bioquímica, 30(2), 41-46.

Cejudo-Ruíz, R., Bautista, F., Quintana, P., DelgadoCarranza, M.C., Aguilar, D., Goguichaishvili, A., Morales-Contreras, J.J., 2015, Correlación entre elementos potencialmente tóxicos y propiedades magnéticas en suelos de la Ciudad de México para la identificación de sitios contaminados: definición de umbrales magnéticos: Revista Mexicana de Ciencias Geológicas, 32 (1), 50-61.

Cortés, J.L., Bautista, F., Quintana, P., Aguilar, D., Goguichaishvili, A., 2015, The color of urban dust as an indicator of contamination by potentially toxic elements: the case of Ensenada, Baja California, Mexico: Revista Chapingo Serie Ciencias Forestales y del
Ambiente, 21 (3), 255-266. https://doi. org/10.5154/r.rchscfa.2015.02.003

Curi, N., Franzmeier, D.P., 1984, Toposequence of Oxisols from the Central Plateau of Brazil: Soil Scince Society of American Journal, 48 (2), 341-346.https://doi.org/10.2136/ sssaj1984.03615995004800020024x

Chen, H., Lu, X., Li, L.Y., Gao, T., Chang Y., 2014, Metal contamination in campus dust of Xi'an, China: A study based on multivariate statistics and spatial distribution: Science of the Total Environment, 484, 27- 35. https:// doi.org/10.1016/j.scitotenv.2014.03.026

Díaz-Rodríguez, J., 2006, Los suelos lacustres de la ciudad de México: Revista Internacional de Desastres Naturales, Accidentes e Infraestructura Civil, 62 (2), 111-129.

Dobos, R.R., Ciolkosz, E.J., Waltman, W.J., 1990, The effect of organic carbon, temperature, time, and redox conditions on soil color: Soil Science and Interdisciplinary Approach to Soils research, 150 (2), 506-512. https://doi. org/10.1097/00010694-199008000-00003

Domínguez Soto, J.M., Román Gutiérrez, A.D., Prieto García, F., Acevedo Sandoval, O., 2012, Sistema de Notación Munsell y CIELab como herramienta para evaluación de color en suelos: Revista Mexicana de Ciencias Agrícolas, 3 (1), 141-155.

Environmental Systems Research Institute Inc (ESRI), 2004, Getting started with ArcGIS 9: Redlands, CA, EUA, Environmental Systems Research Institute Inc.

Fitton, G., 1997, X-ray fluorescence spectrometry, en, Gill, R. (ed.), Modern analytical geochemistry: An introduction to quantitative chemical analysis for earth environmental and materials scientists: Gran Bretaña, Royal Harlow, 87-115.

García, R., Delgado, C., Cejudo, R., Aguilera, A., Gogichaishvili, A., Bautista F., 2019, El color del polvo urbano como indicador de contaminación por metales pesados: Revista Chapingo Serie Ciencias Forestales y del Ambiente, 26 (1), 3-15. https://doi. org/10.5154/r.rchscfa.2019.01.002 
Ihl, T., Bautista, F., Cejudo, R., Delgado, C., Quintana, P., Aguilar, D., Goguitchaichvili, A., 2015, Concentration of toxic elements in topsoils of the metropolitan area of Mexico City: a spatial analysis using Ordinary kriging and Indicator kriging: Revista Internacional de Contaminación Ambiental, 31 (1), 47-62.

Khodeir, M., Shamy, M., Alghamdi, M., Zhong, M., Sun, H., Costa, M., Chen, L., Maciejcczyk, P., 2012, Source apportionment and elemental composition of PM2.5 and PM10 in Jeddah City, Saudi Arabia: Atmospheric Pollution Research 3 (3), 331340. https://doi.org/10.5094/apr.2012.037

Kumaravel, V., Sangode, S.J., Siva Siddaiah, N., Kumar R., 2009, Interrelation of magnetic susceptibility, soil color and elemental mobility in the Pliocene-Pleistocene Siwalik paleosol sequences of the NW Himalaya, India: Geoderma, 154 (2010), 267-280. https:// doi.org/10.1016/j.geoderma.2009.10.013

Levin, N., Ben-Dor, E., Singer, A., 2005, A digital camera as a tool to measure colour indices and related properties of sandy soils in semiarid environments: International Journal of Remote Sensing, 26 (24), 5475-5492. https:// doi.org/10.1080/01431160500099444

Lévy, J., Varela, J., Calvo, A., Rodríguez, M., 2003, Análisis discriminante múltiple, en Lévy, J.P., Varela, J. (coord) Análisis Multivariable para las Ciencias Sociales: Madrid, España, Pearson Prentice Hall, 251-276.

Lozano, R., Bernal, J.P., 2005, Characterization of a new set of eight geochemical reference materials for XRF major and trace element analysis: Revista Mexicana de Ciencias Geológicas, 22 (3), 329-344.

Madeira, J., Bedidi, A., Cervelle, B., Pouget, M., Flay, N., 1997, Visible spectrometric indices of hematite $(\mathrm{Hm})$ and goethite $(\mathrm{Gt})$ content in lateritic soils: the application of a Thematic Mapper (TM) image for soil-mapping in Brasilia, Brazil: International Journal of Remote Sensing, 18 (13), 2835-2852. https://doi.org/10.1080/014311697217369
Marín, P., Sánchez, A., Díaz-Pereira, E., Bautista, F., Romero, M., Delgado, M.J., 2018, Assessment of Heavy Metals and Color as Indicators of Contamination in Street Dust of a City in SE Spain: Influence of Traffic Intensity and Sampling Location: Sustainability, 10, 4105; https://doi. org/10.3390/su10114105

Molina, L.T., Madronich, S., Gaffney, J.S., Apel, E., de Foy, B., Fast, J., Ferrare, R., Herndon, S., Jiménez, J.L., Lamb, B., Osornio-Vargas, A.R., Russell, P., Schauer, J.J., Stevens, P.S., Volkamer, R., Zavala, M., 2010, An overview of the MILAGRO 2006 Campaign: Mexico City emissions and their transport and transformation: Atmospheric Chemistry and Physics, 10, 8697-8760. https://doi. org/10.5194/acp-10-8697-2010

Munsell color, 2000, Munsell ${ }^{\circledR}$ Soil Color Charts: New Windsor, Nueva York. EUA, GretagMacbeth. https://www.worldcat. org/title/munsell-soil-color-charts / oclc/869844188? referer=di\&ht=edition

Paltridge, N.G., Palmer, L.J., Milham, P.J., Guild, G.E., Stangoulis, J.C.R., 2012, Energydispersive X-ray fluorescence analysis of zinc and iron concentration in rice and pearl millet grain: Plant Soil, 361 (1-2), 251-260. https:// doi.org/10.1007/s Res1 1104-011-1104-4

Ramírez Velázquez, B.R., Martínez Reséndiz, J., 2018, La dimensión regional de la movilidad y su impacto en la contingencia ambiental de la Ciudad de México, en Fernández Silva, P. Y., Suárez Lastra, M., Quiroz Rothe, H. (coords), La movilidad en la Ciudad de México. Impactos, conflictos y oportunidades: CDMX, UNAM, 40-55. http://dx.doi.org/10.14350/sc.07

Sabath, E., Robles-Osorio, M.L., 2012, Medio ambiente y riñón: nefrotoxicidad por metales pesados: Nefrología, 32 (3), 279-286. https:// doi.org/10.3265/Nefrologia.pre2012. Jan. 10928

Sánchez-Duque, A., Bautista, F., Goguichaishvili, A., Cejudo-Ruiz, R., Reyes-López, J.A., 
Solís-Domínguez, F.A., Morales-Contreras, J.J., 2015, Evaluación de la contaminación ambiental a partir del aumento magnético en polvos urbanos. Caso de estudio en la ciudad de Mexicali, México: Revista Mexicana de Ciencias Geológicas, 32 (3), 501-513.

Schulze, D.G., Nagel, J.L., Van Scoyoc, G.E., Henderson, T.L., Baumgardner, M.F., Stott, D.E., 1993, The significance of organic matter in determining soil colors, en Soil color, Bigham, M., Ciolkosz, E.J. (ed), Soil Science Society of America,Madison: Wisconsin, EUA, Special Publ, 71-90.

Statgraphics ${ }^{\circledR}$, 2010, Centurion XVI User Manual. Estados Unidos de América, StatPoint Technologies, Inc., 297 p.

Vallejo, M., Jáuregui-Renaud, K., Hermosillo, A.G., Márquez, M.F., Cárdenas, M., 2003, Efectos de la contaminación atmosférica en la salud y su importancia en la Ciudad de
México: Gaceta Médica de México, 139 (1), 57-63.

Vallejo, P.M., 2011, Correlación y regresión simple y múltiple: Madrid, España, Universidad Pontificia Comillas, 24 p.

Viscarra Rossel, R.A., Fouad, Y., Walter, C., 2008, Using a digital camera to measure soil organic carbon and iron contents: Biosystems Engineering, 100, 149-159. https://doi. org/10.1016/j.biosystemseng.2008.02.007

Wei, B., Yang, L., 2010, A review of heavy metal contaminations in urban soils, urban road dusts and agricultural soils from China: MicrochemicalJournal, 94 (2), 99-107. https:// doi.org/10.1016/j.microc.2009.09.014

World Health Organization (WHO), 2014, 7 million premature deaths annually linked to air pollution, (en línea) http://www.who. int/mediacentre/news/releases/2014/ air-pollution/en/12/04/2018 